\title{
Expatriação de atletas e seus aspectos psicológicos: uma revisão sistemática de literatura.
}

\section{Expatriación de atletas y aspectos psicológicos: una revisión de literatura sistemática}

\section{Expatriation of athletes and psychological aspects: a systematic literature review}

\author{
Souza, Vania Hernandes, Miranda, Maria Luiza de Jesus, Correa, Mariana de Freitas, Junior, Marcelo \\ Villas Boas J., Brandão, Maria Regina Ferreira
}

${ }^{1}$ Universidade São Judas Tadeu

Financiamento:

CAPES - concessão de bolsa para Vania Hernandes de Souza (88882.366392 / 2019-01)

\section{RESUMO}

Atletas de modalidades esportivas migram ao longo de suas carreiras para outros países em busca de diferentes objetivos, dentre os quais alcançar melhor desempenho e status sociais e financeiro. Nesse sentido, o objetivo deste estudo foi revisar a literatura em relação aos aspectos psicológicos envolvidos no processo de expatriação de atletas. Para tanto, foram realizadas buscas nas bases de dados Scielo, PubMed, SportDiscus, BVS, Embase e Banco de teses e dissertações da CAPES, com vocábulos próprios de cada base, nos idiomas inglês, português e espanhol. Foram incluídos estudos que contemplassem atletas de rendimento e investigassem o processo de migração internacional no esporte, além de excluídos os estudos de revisão e estudos realizados com estudantes, atletas estudantes e técnicos. Após criteriosa triagem, realizada por meio do programa gerenciador de referências bibliográficas, o software EndNote ${ }^{\circledR}$, foram selecionados e armazenados oito estudos elegíveis para análise, utilizando o modelo temporal de transição cultural. Assim, os resultados obtidos ilustraram que, entre os atletas, não é uma prática comum a busca por informações prévias sobre o país hospedeiro e/ou o contexto esportivo em que serão inseridos. Já, em relação ao processo de adaptação cultural aguda, evidenciou-se que a principal estratégia utilizada, ligada às necessidades psicológicas básicas, somada ao aprendizado do idioma, foram fundamentais na promoção da sensação de pertencimento à cultura e, consequentemente, o desenvolvimento da adaptação sociocultural. Diante disso, concluise que investigar os aspectos psicológicos envolvidos na migração internacional de atletas, oferecendo o preparo psicológico para enfrentar os desafios pertinentes à adaptação cultural, provavelmente contribuirão para o melhor entendimento nas escolhas das estratégias a serem utilizadas nesse processo, bem como podem ser determinantes para o sucesso da migração.

Palavras-chave: esportes, atletas, aspectos psicológicos e migração internacional. 


\title{
Souza, Miranda, Correa, Junior \& Brandão
}

\section{RESUMEN}

Los atletas de modalidades deportivas migran a lo largo de su carrera a otros países en busca de diferentes objetivos, entre ellos, para lograr un mejor rendimiento y un estado social y financiero. En este sentido, el objetivo de este estudio fue revisar la literatura en relación con los aspectos psicológicos involucrados en el proceso de expatriación de los atletas. Para este fin, se realizaron búsquedas en las bases de datos de tesis y disertaciones de Scielo, PubMed, SportDiscus, BVS, Embase y CAPES, con palabras específicas para cada base, en inglés, portugués y español. Se incluyeron estudios que incluyeron atletas de rendimiento e investigaron el proceso de migración internacional en el deporte y se excluyeron los estudios de revisión y estudios con estudiantes, estudiantes atletas y entrenadores. Después de una cuidadosa selección, realizada a través del programa administrador de referencias bibliográficas, el software EndNote ${ }^{\circledR}$, se seleccionaron y almacenaron ocho estudios elegibles para el análisis utilizando el modelo temporal de transición cultural. Por lo tanto, los resultados obtenidos ilustraron que, entre los atletas, no es una práctica común buscar información previa sobre el país anfitrión y / o el contexto deportivo en el que se insertarán. En relación con el proceso de adaptación cultural aguda, se hizo evidente que la estrategia principal utilizada está vinculada a las necesidades psicológicas básicas, además del aprendizaje de idiomas, fueron fundamentales para promover el sentimiento de pertenencia a la cultura y, en consecuencia, el desarrollo de Adaptación sociocultural. Por lo tanto, se concluye que investigar los aspectos psicológicos involucrados en la migración internacional de atletas, ofreciendo la preparación psicológica para enfrentar los desafíos pertinentes a la adaptación cultural, probablemente contribuirá a una mejor comprensión de las opciones de estrategias que se utilizarán en este proceso, así como determinando el éxito de la migración.

Palabras-clave: deportes, atletas, aspectos psicológicos, migración internacional.

\begin{abstract}
Athletes from sports modalities migrate throughout their career to other countries in search of different objectives, among them, to achieve better performance and social and financial status. In this sense, the objective of this study was to review the literature in relation to the psychological aspects involved in the expatriation process of athletes. For this purpose, searches were carried out in the Scielo, PubMed, SportDiscus, BVS, Embase and CAPES theses and dissertations databases, with words specific to each base, in English, Portuguese and Spanish. Studies that included performance athletes and investigated the process of international migration in sport were included and review studies and studies with students, student athletes and coaches were excluded. After careful screening, carried out by means of the bibliographic reference manager program, the EndNote ${ }^{\circledR}$ software, eight studies eligible for analysis were selected and stored using the temporal model of cultural transition. Thus, the results obtained illustrated that, among athletes, it is not a common practice to search for previous information about the host country and / or the sports context to which they will be inserted. In relation to the process of acute cultural adaptation, it became evident that the main strategy used is linked to basic psychological needs, in addition to language learning, were fundamental in promoting the feeling of belonging to culture and, consequently, the development of sociocultural adaptation. Therefore, it is concluded that investigating the psychological aspects involved in the international migration of athletes, offering the psychological preparation to face the challenges pertinent to cultural adaptation, will probably contribute to a better understanding in the choices of strategies to be used in this process, as well as can determining the success of the migration.
\end{abstract}

Keywords: sports, athletes, psychological aspects, international migration. 


\section{Expatriação de atletas e seus aspectos psicológicos}

\section{INTRODUÇÃO}

Devido à globalização do esporte, que facilita a migração de atletas quanto ao deslocamento e a questões burocráticas, a cada ano o número de atletas de diferentes modalidades esportivas que joga em outro país que não o seu de origem chega aos milhares, seja com o objetivo de constituir e consolidar a carreira esportiva (Faggiani et al., 2016) ou por melhoria de vida e aumento salarial (Tertuliano, Machado, Deutsch, Montiel, \& Bartholomeu, 2018).

Como exemplo dessa globalização, tem-se o futebol, com efetivas mudanças do mercado de transferências dos atletas, sendo o Brasil um dos principais protagonistas (Brandão, Magnani, Medina, \& Tega, 2013). De acordo com o relatório produzido pela Diretoria de Registro, Transferência e Licenciamento da Confederação Brasileira de Futebol (CBF), no ano de 2019, o Brasil contabilizou a saída de 1.464 jogadores para o mundo. Outros exemplos podem ser observados entre as equipes Europeias, tais como a do PSG da França, que conta no seu plantel com jogadores de doze países diferentes ou a equipe do Barcelona da Espanha, que contabiliza 11 jogadores de diferentes nacionalidades.

Tal fato se repete em outras modalidades esportivas: no voleibol, o time Trentino Volley da Itália tem no plantel principal de 2020 jogadores de cinco países diferentes; no handebol feminino, a equipe Gyori da Hungria é formada por atletas de quatro países diferentes e na liga norte-americana masculina de basquetebol, a NBA (National Basketball Association), o número de jogadores nascidos fora do país, na temporada 2018/2019, somaram 108, representando 38 nações.

Devido ao crescente número de atletas expatriados pelo mundo, o fenômeno de migração ou transição migratória no esporte tem despertado a atenção no contexto acadêmico (Schwartz, Unger, Zamboanga, \& Szapocznik, 2010), impulsionando investigações sobre as vias de adaptação ou aculturação nos países de acolhimento (Ryba, Schinke, Stambulova, \& Elbe, 2017), a relação entre fatores psicológicos e culturais no processo de adaptação cultural (Agergaard \& Ryba, 2014), a identificação dos desafios encontrados na migração, além da proposta de estratégias para acelerar o processo de adaptação do atleta migrante
(Schinke, Yukelson, Bartolacci, Battochio, \& Johnstone, 2011).

A atenção em torno da aculturação de atletas migrantes ou expatriados ocorre porque a migração é estudada como um processo de mudança de carreira (Agergaard \& Ryba, 2014) e, no campo da psicologia esportiva, as experiências oriundas da migração são definidas como um processo de mudança cultural e psicológica, em identidade, práticas e valores, resultantes do contato regular e exclusivo entre pessoas de diferentes grupos sociais (Schwartz et al., 2010)

Assim, observa-se que, para o atleta expatriado, tais mudanças podem ser consideradas complexas, pois envolvem não apenas o desafio de morar em um novo país e se adaptar a uma nova cultura que pode diferir em termos de alimentação, idioma e clima, mas também à convivência e adaptação a uma nova equipe, a um treinador desconhecido (Yukelson \& Carlson, 1996), ao nível elevado de competição e expectativas de desempenho rigorosas da equipe de treinamento, a colegas de equipe e ao público (Hanton, Fletcher \& Coughlan, 2005).

Quanto aos desafios encontrados para a adaptação e o ajuste em um novo contexto, (Yukelson \& Carlson, 1996) pontuam que podem afetar a concentração e os sentimentos de autoestima dos atletas. Para Brandão et al., (2013, pp.177-182), "a habilidade para se adaptar a novas culturas é um dos elementos mais importantes para o sucesso de um jogador de futebol [...]". Igualmente, a necessidade de se estabelecer e adaptar prontamente às novas demandas sociais pode afetar a saúde mental e o desempenho esportivo devido à ruptura com a vida cotidiana e o significado interno da experiência (Magnani, 2018).

Diante do exposto, as investigações envolvendo atletas migrantes recai sobre o processo de transição de carreira, no sentido de identificar como enfrentaram a adaptação e aculturação ao novo país, bem como os obstáculos, os recursos e as estratégias utilizadas para facilitar uma transição bem-sucedida em um novo ambiente esportivo (Agergaard \& Ryba, 2014).

É interessante notar, entretanto, que apesar de toda a complexidade envolvida nesse processo, a migração esportiva só foi documentada na literatura das ciências do esporte ao longo dos últimos 20 anos (Diakaki, 


\section{Souza, Miranda, Correa, Junior \& Brandão}

Chroni \& Goudas, 2016). Inicialmente, ela foi investigada como um fenômeno sociológico (Maguire, 1994), visto que a atenção dada a uma vertente de ordem psicológica é relativamente recente (Schinke, et al., 2011; Ryba, Haapanen, Mosek, \& Ng, 2012).

Para Ryba et al., (2017), com o ritmo historicamente sem precedentes da internacionalização da indústria esportiva e do movimento transnacional de talentos esportivos, houve a necessidade de desenvolvimento de novas competências na pesquisa e na prática diária dos profissionais da psicologia do esporte. Além disso, acrescentam que, enquanto a literatura acadêmica em psicologia e práxis do esporte cultural vem aumentando, os profissionais do esporte $\mathrm{e}$ as organizações locais parecem dar pouco tempo e recursos para se manterem a par das complexas mudanças sociais na indústria transnacional e para $o$ desenvolvimento de competências culturais.

Assim, o objetivo deste estudo foi realizar uma revisão sistemática da literatura em relação aos aspectos psicológicos envolvidos no processo de adaptação e aculturação internacional de atletas expatriados.

\section{MATERIAL E MÉTODOS}

Foi realizada uma revisão sistemática de literatura qualitativa, sem restrição de período de publicação, que seguiu os procedimentos recomendados por (Galvão \& Pereira, 2014; Marconi \& Lakatos, 2012) para pesquisas dessa natureza e teve a finalidade de identificar, qualificar e sintetizar os estudos relacionados aos aspectos psicológicos envolvidos na expatriação de atletas.

Esta revisão iniciou-se no dia 27 de setembro de 2019, com a elaboração da pergunta norteadora para a busca de evidências, utilizando o formato PICO (Stone 2004), cujos quatro componentes (figura1) são os elementos fundamentais da questão de pesquisa e da construção da pergunta para a busca bibliográfica (Santos, Pimenta \& Nobre, 2007), sendo definida a seguinte pergunta: "Quais os aspectos psicológicos envolvidos no processo de adaptação e aculturação de atletas?".
Descrição da estratégia PICO

Tabela 1 Descrição da estratégia PICO

\begin{tabular}{cc}
\hline Itens PICO & Descrição \\
\hline População & Atletas \\
\hline Intervenção & $\begin{array}{c}\text { Processo de } \\
\text { Expatriação }\end{array}$ \\
\hline Comparação & \\
\hline Outcomes & $\begin{array}{c}\text { Impacto } \\
\text { Psicológico }\end{array}$ \\
\hline
\end{tabular}

\section{Procedimiento}

Definida a problemática do estudo, foi realizada pesquisa nos descritores da saúde DeCS, Mesh Terms e Emtree e um levantamento bibliográfico retrospectivo pelas seguintes bases de dados: Scielo, PubMed, SportDiscus, BVS e Embase utilizando os booleanos " $A N D$ " $e$ "OR" e os termos específicos (Athletes OR Athlete OR Sports OR Athletic OR Athletics OR Sport) AND (Human Migration OR Human Migrations OR Migration, Human OR Migrations, Human OR International Migration OR International Migrations OR Labor Migration OR Labor Migrations OR Migration, International OR Migration, Labor OR Migrations, International OR Migrations, Labor OR Migrations, Temporary OR Temporary Migration OR Temporary Migrations OR Migrant Athletes OR Transnational mobility), espanhol: (Atletas OR Deportes OR Actividades Deportivas) AND (Migración OR Migración Humana OR Migración Internacional OR Migración Laboral OR Migración de Ocupación) e português: (Atleta OR Esportes OR Atividades Esportivas OR Esportes OR Desportos) e todas as combinações prováveis, utilizando o delimitador " $A N D$ ", nas bases de dados Scielo, PubMed, SportDiscus, Portal BVS e Embase. Igualmente, foi adicionada à pesquisa a busca pelo Banco de Teses e Dissertações da CAPES, através dos termos Migração Esportiva, Expatriação Esportiva, Aculturação e Esporte.

Ao fim da etapa de busca, os estudos encontrados foram exportados para o software EndNote X9®, um gerenciador de referências, que, entre os seus recursos, estão o de pesquisar em bases de dados diretamente do EndNote Web, detectar estudos duplicados e pesquisar em fontes como o Scopus, Medline e Google Acadêmico e importar os registros para o EndNote 


\section{Expatriação de atletas e seus aspectos psicológicos}

Web (Yamakawa, Kubota, Beuren, Scalvenzi, \& Miguel, 2014).

A triagem dos estudos iniciou-se com a identificação e eliminação, por meio do software EndNote X9® e manualmente, dos estudos duplicados. Em seguida, seguindo os procedimentos sugeridos por Pilkington \& Hounsome (2017), ocorreu a leitura do título e resumo e o descarte dos artigos aplicando os critérios de inclusão e exclusão.

Como critérios de inclusão, foram definidos estudos publicados nos idiomas inglês, espanhol e português, estudos na íntegra e que apresentavam a temática relativa a essa revisão sistemática, estudos que continham atletas em sua amostra e que investigavam o processo de expatriação no esporte. Aos critérios de exclusão, foram definidos estudos de revisão e estudos cuja amostra era composta por estudantes, estudantes/atletas e técnicos.

Por fim, ainda seguindo os procedimentos de Pilkington \& Hounsome (2017), realizou-se a leitura completa e detalhada dos estudos incluídos, utilizando-se dos mesmos critérios de inclusão e exclusão. As etapas estão ilustradas no fluxograma da figura 1 .

A extração dos dados foi dividida em resultados descritivos (tabela 2) e resultados analíticos (tabela 3). A análise do conteúdo foi realizada de acordo com as orientações de (Olabuénaga \& Ispizua, 1989) e, para a definição das informações analíticas dos participantes do estudo, utilizou-se o método indutivo, tendo como base o Modelo Temporal de Transição Cultural de Ryba, Stambulova, and Ronkainen (2016) descrito a seguir.

De acordo com o modelo, a análise do processo de adaptação e aculturação delineia-se em três fases: prétransição, adaptação cultural aguda e adaptação sociocultural, todas com tarefas de desenvolvimento específicas. Igualmente, o modelo propõe três mecanismos subjacentes que realizam a transição e modelam os caminhos que podem ser tomados: reposicionamento social em redes translocais, negociação de práticas culturais e reconstrução de significados.

A fase de pré-transição refere-se à fase anterior ao processo de migração internacional, ocorre dentro do país de origem ou onde o atleta está locado, terminando apenas quando o atleta chega fisicamente ao novo país. Essa fase visa a preparação, por meio da coleta de informações relevantes sobre o país de migração, para a realocação dos atletas.

Já na fase de adaptação cultural aguda, ocorre o entendimento e o ajuste à nova cultura, podendo, dessa forma, ocasionar o choque cultural, pois trata-se do momento da adaptação psicológica e comportamental. Essa fase é dividida em adaptação geral, adaptação ao clube e adaptação à interação, e tem-se como expectativa que o atleta se adapte às demandas, expectativas e diferenças culturais do ambiente de treinamento desconhecido.

Por fim, na fase de adaptação sociocultural ocorre o ajustamento a nova realidade, ou seja, é momento de contato e interação do atleta com o clube, os companheiros de equipe e a nova cultura, proporcionando um sentimento de pertencimento. Essa fase ocorre a longo prazo e revela que o atleta superou algumas das dificuldades vividas no processo de adaptação aguda. Ainda, segundo Ryba et al. (2016) é nessa fase em que ocorre o ajuste psicológico, isto é, o atleta mantém-se no país por um período maior ou opta por se estabelecer nesse país em definitivo. 


\section{Souza, Miranda, Correa, Junior \& Brandão}

Figura 1. Fluxograma da Revisão Sistemática

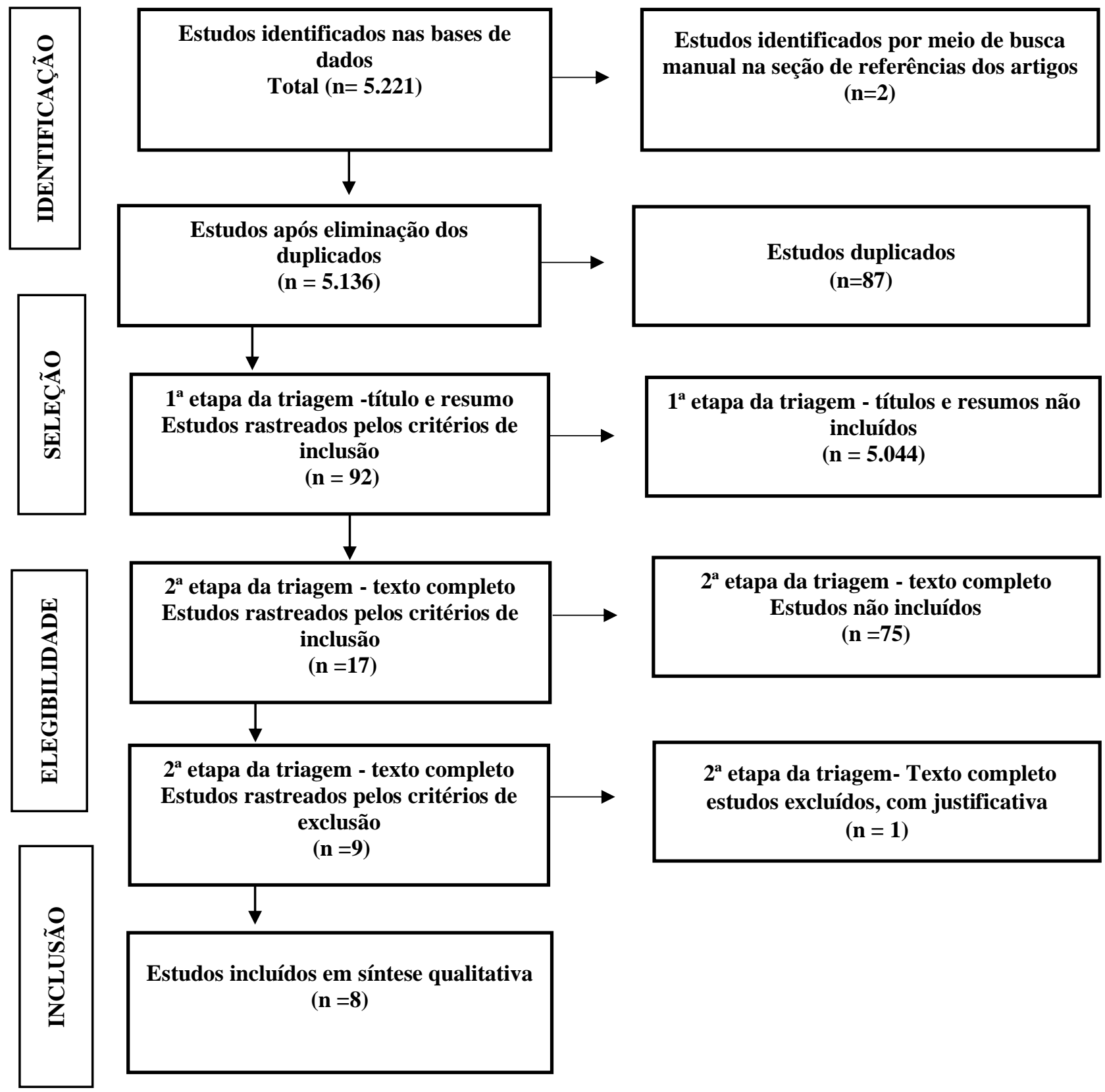




\section{Expatriação de atletas e seus aspectos psicológicos}

\section{RESULTADOS}

Após a realização das buscas nas bases de dados e do processo de triagem de todos os estudos, foram incluídos oito estudos para análise. Os estudos incluídos estão apresentados na tabela 2, composta dos dados descritivos, apresentados da seguinte maneira: autores, ano, publicação, objetivo, participantes (atletas), idade (anos), modalidade esportiva e instrumento utilizado. Os resultados analíticos são descritos na (tabela 3), que inclui os resultados da conclusão.

\section{DISCUSSÃO}

A análise descritiva mostrou que os estudos selecionados envolvendo a migração atlética concentram-se no continente europeu, e que apenas dois foram realizados no Brasil. Entretanto, no Brasil, as investigações sobre a temática têm avançado por meio das pesquisas voltadas à modalidade futebol Brandão et al., (2013) e Brandão and Vieira (2013) que investigaram o impacto psicológico referente ao processo de adaptação cultural no contexto esportivo.

Ainda, sobre a temática migração atlética, constatouse um intervalo de seis anos entre os estudos de (Schinke et al., 2006) e o estudo de (Ryba et al., 2012). Quanto a essa constatação, é importante esclarecer que na investigação realizada nessa revisão sistemática foram encontrados estudos nesse intervalo, mas eles eram voltados à área da sociologia esportiva (Magee \& Sugden, 2002; Maguire, 2004), visto que apenas no ano de 2009 a temática passou também a ser analisada sob a ótica da psicologia do esporte (Schinke, McGannon, Battochio, \& Wells, 2013).

Em relação aos demais estudos encontrados nesse período, eles não atendiam aos critérios de inclusão estabelecidos para essa revisão sistemática, tais como: os artigos de revisão de Vasconcellos Ribeiro and Dimeo (2009) e Botelho and Agergaard (2011), o estudo sobre a análise do aumento do comércio de trabalho no futebol africano e o estabelecimento de academias de futebol na África, ocasionadas pela migração atlética (Darby, Akindes, \& Kirwin, 2007) e o uso do esporte como instrumento de integração de imigrantes nos Estados Unidos para tirar o emprego dos americanos (Borgese, 2009).

Quanto aos instrumentos, foi possível identificar que 7 estudos utilizaram a entrevista semiestruturada e um estudo, a entrevista não estruturada. A base das perguntas foi direcionada no sentido de: investigar quais foram os desafios oriundos da adaptação a um novo ambiente (estudo 1), explorar a dinâmica associada aos aspectos psicológicos e sociais da adaptação cultural aguda (estudo 2), questões-chave pertinentes à aculturação de atletas imigrantes (estudo 3), migração transnacional e desenvolvimento de carreira esportiva (estudo 4), transição de carreira, migração, deslocamento e identidade vocacional (estudo 5), migração e identidade (estudo 6), adaptação cultural e os aspectos relacionados e influenciadores no desempenho esportivo e a percepção sobre o processo de migração (estudo 7) e questões pertinentes ao Modelo Temporal de Transição Cultural (estudo 8).

A respeito das modalidades esportivas descritas, houve um equilíbrio entre as praticadas de forma individual e coletiva, mas os estudos analisados nessa revisão não apontaram se o processo de adaptação e aculturação estão intimamente relacionados ao tipo de modalidade esportiva. No entanto, observou-se que os atletas migrantes do estudo 1, na condição de praticantes de esportes coletivos e pertencentes ao mesmo país de origem, relataram a necessidade de confiar e se apoiar nos conterrâneos a fim de aceitarem o novo ambiente e receberem suporte.

Igualmente, as atletas do estudo 2, também migrantes na mesma condição dos atletas do estudo 1, mas praticantes de esporte individual, afirmaram que, mesmo não praticando uma modalidade esportiva coletiva, é preciso ter o apoio dos colegas conterrâneos da equipe. E, a exemplo dos atletas do estudo 1, elas também se uniram para vencer o principal desafio enfrentado: a barreira da comunicação.

No caso dos dois estudos, pelo fato de os atletas serem da mesma nacionalidade e viajarem em equipe, houve uma tendência de se unirem e buscarem apoio junto aos colegas do mesmo país (Soler, 2020) e em nível de identificação, esse grupo pode ser uma rede de segurança que favorece o pertencimento e o desenvolvimento atlético (Ryba et al., 2012). 
Cuadernos de Psicología del Deporte, vol. 21, 1, 119-134

Recibido: 27/06/2020

Aceptado: 30/09/2020
(C) Copyright 2018: Servicio de Publicaciones de la Universidad de Murcia Murcia (España) ISSN edición impresa: 1578-8423 ISSN edición web (http://revistas.um.es/cpd): 1989-5879
No que concerne ao esporte praticado individualmente Soler (2020) coloca que cada um tem as suas particularidades e cada atleta deve ser estudado de forma isolada, mas, como habitualmente acontece, o atleta sempre estará rodeado pelo seu treinador, que irá inserir pessoas de diferentes países na sua integração. Nesse sentido, a autora indica que a adaptação pode ocorrer porque, ao ser por natureza social, o ser humano tende a se unir uns aos outros.

No que diz respeito ao papel do treinador no processo de adaptação, o estudo de Oliveira, Dias, Sabino, Corte-Real, Maia \& Fonseca (2019), ilustra a importância do seu suporte social para oportunizar o desenvolvimento e a satisfação dos atletas. Provavelmente, ao oferecer esse ambiente de suporte, os atletas vivenciarão experiências afetivas positivas que resultarão em elevados níveis de motivação, bemestar e persistência (Occhino, Mallett, Rynne, \& Carlisle, 2014), facilitando assim, a adaptação ao novo ambiente.

A amostra dos estudos contou com 58 atletas do sexo masculino e 43 atletas do sexo feminino, na faixa etária entre 16 a 42 anos, mas nenhum estudo mencionou ou teve como objetivo investigar aspectos ligados ao sexo e à faixa etária no processo de migração atlética.

Para a discussão dos resultados analíticos, utilizou-se o Modelo Temporal de Transição Cultural, em que cada fase é importante por apontar as tarefas de desenvolvimento que facilitam ou não a adaptação e/ou aculturação (Ryba et al., 2016).

Portanto, na fase de pré-transição, em que se obtém ainda no país de origem informações sobre a cultura hospedeira, dá-se início a um processo de desapego, com a desvinculação de pessoas, família e lugares e início ao desenvolvimento de vínculos com a nova cultura. Igualmente, as informações adquiridas nessa fase preparam o atleta mentalmente para os desafios existentes em uma transição cultural (Lofredo, 2018).

Dada a importância dessa fase no processo de transição e mesmo com o acesso às tecnologias de informação ou aos recursos tecnológicos, apenas no estudo 5 os atletas buscaram informações prévias sobre o país e o contexto esportivo a ser inserido.
Contudo, não foram mencionadas como as informações foram adquiridas, quais informações eram relevantes e/ou consideradas facilitadoras no processo de preparação mental, no ajuste com a equipe e com a cultura local, bem como se as informações, ou a falta delas, influenciaram de alguma forma na fase seguinte.

A fase de adaptação cultural aguda é provavelmente a fase mais importante de todo processo, pois ocorre o entendimento e o ajuste a nova cultura, podendo, assim, ocasionar o choque cultural. Nessa fase, os estudos relataram que idioma, clima, alimentação, religião, regras de convivência, normas culturais locais, valores, estilos de vida e de trabalho da nova equipe e interação com o treinador e companheiros (as) de equipe podem determinar o sucesso ou não da experiência migratória.

Ryba et al., (2016) esclarecem que esta fase não está associada apenas aos processos de adaptação individual a um novo ambiente sociocultural, mas também aos processos de manutenção de ligações sociais com familiares, amigos e organizações na sua sociedade de origem, percebidas nos relatos do estudo 6 , cujos sentimentos de solidão e tristeza, causados pelo afastamento da família, amigos e do povo, ocasionaram a desistência do atleta.

Quanto às principais estratégias de adaptação, os estudos 2 e 8 evidenciaram a negociação das práticas cotidianas como primordiais para manter o bem estar psicológico e alcançar as necessidades psicológicas básicas. $\mathrm{O}$ ajuste a essas práticas cotidianas é explicado pelo modelo como um processo aberto, de negociações entre manter uma sensação subjetiva de bem-estar e participar de práticas cotidianas aculturadoras Ryba et al., (2012), além de enfatizar a capacidade de encaixar-se e tornar-se culturalmente fluente em um novo contexto (Ward \& Kennedy, 1999).

Para alcançar o bem estar psicológico e não perder o senso de si próprio, conforme relatado no estudo 5, os atletas buscaram o comprometimento e a integração com a equipe, o aprendizado sobre a cultura, a construção de relações com os membros da equipe, a evolução do próprio entendimento sobre a cultura no contexto de prática e da vida social no país anfitrião, a capacidade de superação ao se deparar com 


\section{Expatriação de atletas e seus aspectos psicológicos}

preconceitos, a humilhação e falta de apoio, ilustrados pelas atletas do estudo 1 e 8 , e ser resiliente diante das adversidades, observado no estudo 2 .

As dificuldades de livrar-se do preconceito de pertencer a uma cultura já marginalizada, de se adaptar às regras e estruturas organizacionais, de integrar-se à nova equipe e solucionar os problemas de relacionamento com o técnico e as atletas, relatados nos estudos 1, 6, 7 e 8 , foram superados por meio de estratégias adaptativas que os indivíduos usaram em resposta a novos contextos culturais e que estão ligadas ao desenvolvimento da identidade (Berry \& Padilla, 1980), construída a partir do significado interno das experiências vividas na nova rotina, e que possibilita ao atleta tomar consciência dos padrões culturais e da sua identidade cultural (Brandão et al., 2013), iniciando, assim, o processo de pertencimento à nova cultura.

Em relação à identidade, para um atleta do estudo 6, a mudança para um ambiente diferente e desconhecido desencadeou a frustração pela perda das relações pessoais e das várias tentativas de tentar se integrar, adaptar e reestruturar o seu senso de identidade. Nesse sentido, a identidade atlética refere-se à identificação com o seu papel e à sua autovalorização (Brewer, Van Raalte, \& Linder, 1993). O que ilustra a importância dos significados dados aos múltiplos papéis que a identidade pode ter (Oyserman, Elmore \& Smith, 2012), principalmente a do atleta, que é influenciada pelo ambiente social (Stoltenburg, Kamphoff, \& Bremer, 2011).

Quanto ao sentimento de pertencimento, este manifesta-se também por meio da aceitação dos atletas pela equipe anfitriã, relatados no estudo 1 , e, consequentemente, da construção de relacionamentos, importantes não apenas para o sucesso da adaptação cultural do atleta, mas também da equipe. Nesse sentido, é importante considerar que as estratégias de adaptação apoiadas devem incluir o eu e o outro, visto que a adaptação é um processo dinâmico da negociação entre manter o equilíbrio psicológico e o envolvimento nas práticas socioculturais no país anfitrião (Schinke et al., 2006).

Para os migrantes engajados ativamente em redes e atividades transnacionais, a pertença transnacional é essencial para a formação de identidade e dos autoconceitos (Ryba et al., 2016) e, provavelmente, podem favorecer $\mathrm{o}$ processo de adaptação sociocultural.

No estudo 5, a satisfação, tanto com o ambiente esportivo quanto com a sociedade em geral, pode ser observada nos relatos dos atletas em relação ao sentimento de compatibilidade entre os próprios valores e estilos de vida, com as normas culturais locais, identificadas por meio da vida cotidiana em vários contextos sociais. Nessa perspectiva, a satisfação pode ser entendida como um domínio bem sucedido do contexto esportivo e da cultura do país de acolhimento (Ryba et al., 2012), resultante da adaptação psicológica, voltada ao bem-estar mental e da adaptação sociocultural, que destaca a habilidade de inserção e fluência em um novo contexto cultural (Ward \& Kennedy, 1999).

Sobre o sentimento de compatibilidade, ele pode ter ocorrido porque os atletas conseguiram equilibrar de forma harmoniosa o valor dado ao desenvolvimento da carreira e às questões pertinentes a outros domínios da vida, tais como atividades sociais e lazer, importantes para a adaptação social, como apontado no estudo de (Tretyakevich, Las Heras, \& Hall, 2015).

$\mathrm{O}$ aspecto comunicação também foi mencionado no processo de adaptação sociocultural e é entendida tanto como desafiadora (Schinke, et al., 2011), quanto facilitadora das conexões (Battochio et al., 2013). Nos estudos 7 e 8 , a comunicação foi facilitada devido à semelhança do idioma, e sua importância foi pontuada como um diferencial nesse processo. Contudo, a comunicação é uma barreira, como mencionado no estudo 2, quando ocorre a ausência de uma linguagem comum de comunicação (Diakaki, Chroni \& Goudas, 2016).

\section{CONCLUSÃO}

Essa revisão buscou investigar os aspectos psicológicos envolvidos no processo de adaptação e aculturação internacional de atletas expatriados à luz do Modelo Temporal de Transição Cultural e apontou um reduzido número de estudos voltados à temática, por isso demandam um maior aprofundamento sobre o assunto. 


\section{Souza, Miranda, Correa, Junior \& Brandão}

Quanto às variáveis investigadas, os resultados mostraram que não é uma prática comum entre os atletas obter informações culturais sobre o país hospedeiro ou sobre o contexto de prática a que está se inserindo. Nesse sentido, a falta de preparo na fase de pré-transição dificultou a realocação e, provavelmente, pode ser agravada pela distância da família, dos amigos e do convívio social ao qual o atleta estava acostumado.

$\mathrm{Na}$ fase de preparação cultural aguda, ficou claro que ela está associada ao desenvolvimento das habilidades psicológicas básicas e à busca do equilíbrio entre o bem-estar psicológico e o envolvimento nas atividades socioculturais. Entretanto, é importante considerar que o tempo de permanência, bem como os objetivos de cada atleta, também influenciaram nessa adaptação.

Outro aspecto a ser considerado foi a de que nenhum estudo apontou apoio psicológico, por parte dos contextos esportivos, para receber os atletas migrantes e auxiliar na adaptação ao novo ambiente cultural. Diante disso, observou-se que cada atleta utilizou uma ferramenta para facilitar a sua adaptação e superar os desafios pessoais, profissionais e a barreira do idioma.

Quanto à adaptação sociocultural, identificou-se que a aprendizagem compartilhada de comunicação e a troca de experiências e aprendizado sobre as culturas dos atletas migrantes foi um diferencial positivo nessa fase do processo. Nesse aspecto, quando o atleta busca vencer as dificuldades de adaptação por meio do envolvimento com a nova cultura, inclusive na tentativa de aprender o novo idioma, tem-se um processo de adaptação social.

Apesar da constatação de que há um considerável número de atletas migrantes, observou-se a importância e, consequentemente, a necessidade de um melhor preparo, desde a fase de pré-transição, no sentido de minimizar os impactos psicológicos causados pela brusca mudança de padrões de vida, cultura, métodos e sistemas de treinamento.

\section{APLICAÇÕES PRÁCTICAS}

Acredita-se que investigar os aspectos psicológicos envolvidos no fenômeno migração internacional de atletas e oferecer o preparo psicológico para o enfrentamento dos desafios pertinentes ao processo de adaptação cultural, contribuirão para o melhor entendimento sobre o tema e, consequentemente, os auxiliarão nas escolhas das estratégias adequadas a serem utilizadas em todas as fases descritas pelo Modelo de Transição Cultural. Igualmente, é importante que as pessoas envolvidas nesse processo tomem posse desse conhecimento para entender como devem intervir com os atletas migrantes a fim de facilitar a adaptação.

\section{REFERÊNCIAS}

1. Agergaard, S., Ryba, T. V. (2014). Migration and career transitions in professional sports: Transnational athletic careers in a psychological and sociological perspective. Sociology of Sport Journal, $\quad 31(2), \quad 228-247$. https://doi.org/10.1123/ssj.2013-0031.

2. Battochio, R. C., Schinke, R. J., McGannon, K. R., Tenenbaum, G., Yukelson, D., Crowder, T. (2013). Understanding immigrated professional athletes' support networks during post-relocation adaptation through media data. International Journal of Sport and Exercise Psychology, 11(1), 101-116.

https://doi.org/10.1080/1612197X.2013.748996.

3. Berry, J. W., Padilla, A. M. (1980). Acculturation: Theory, models and some new findings. Acculturation as varieties of adaption. Boulder: Westview Press

4. Borgese, A. (2009) Labor and immigration issues in sports. Sport Journal, 12(3), 1-12.

5. Botelho, V. L., Agergaard, S. (2011). Moving for the love of the game? International migration of female footballers into Scandinavian countries. Soccer \& Society, (12) 6 806-819. https://doi.org/10.1080/14660970.2011.609681.

6. Brandão, M. R. F., Magnani, A., Medina, J. P., Tega, E. C. (2013). Além da cultura nacional: o expatriado no futebol. Revista Brasileira de Ciência e Movimento, 21(2), 177-182.

7. Brandão, M. R. F., Vieira, L. F. (2013). Athletes' careers in Brazil: Research and application in the land of ginga. Stambulova, N. B., Ryba, T. V. (Eds.), Athletes' careers across cultures, 63-72. Abingdon, UK: Routledge.

8. Brewer, B. W., Van Raalte, J. L., Linder, D. E. (1993). Athletic identity: Hercules' muscles or 


\section{Expatriação de atletas e seus aspectos psicológicos}

Achilles heel? International journal of sport psychology, 24(2), 237-254.

9. Darby, P., Akindes, G., Kirwin, M. (2007). Football academies and the migration of African football labor to Europe. Journal of sport and social issues, 31(2), 143-161. https://doi.org/10.1177/0193723507300481.

10. Diakiki, E., Chroni, S. A., Goudas, M. (2016). Migrating cyclists and identity reconstructions: Two tales of living and parting the dream. Inquiries in Sport \& Physical Education 14(3), 87-101.

11. Faggiani, F., Lindern, D., Strey, A., Aiquel, P. F., Fulginiti, D., Sartori, C., Lisboa, C. S. d. M. (2016). O fenômeno do expatriado no contexto esportivo. Psicologia: Ciência e Profissão, 36(3), 738-747. http://dx.doi.org/10.1590/1982-3703001832016.

12. Galvão, T. F., Pereira, M. G. (2014). Revisões sistemáticas da literatura: passos para sua elaboração. Epidemiologia e Serviços de Saúde, 23(1), 183-184. http://dx.doi.org/10.5123/S167949742014000100 018.

13. Hanton, S., Fletcher, D., Coughlan, G. (2005). Stress in elite sport performers: A comparative study of competitive and organizational stressors. Journal of sports sciences, 23(10), 1129-1141. https://doi.org/10.1080/02640410500131480.

14. Lofredo, M.C. (2018). Migração e aculturação de atleta de elite do handebol feminino brasileiro: Estudo de caso (Dissertação de Mestrado). Universidade São Judas Tadeu, USJT, São Paulo, SP, Brasil. Disponível em: http://cev.org.br/biblioteca/migracao-e aculturacao-de-atleta-de-elite-do-handebolfeminino-brasileiro-estudo-de-caso. Acesso em: 10 set. 2020.

15. Magee, J., Sugden, J. (2002). "The World at their Feet" Professional Football and International Labor Migration. Journal of sport and social issues, 26(4), 421-437. https://doi.org/10.1177/0193732502238257.

16. Maguire, J. (2004). Sport labor migration research revisited. Journal of sport and social issues, 28(4), 477-482. https://doi.org/10.1177/0193723504269914.

17. Marconi, M. D. A., Lakatos, E. M. (2012). Técnicas de pesquisa: planejamento e execução de pesquisa; amostragens e técnicas de pesquisa; elaboração, análise e interpretação de dados Técnicas de pesquisa: planejamento e execução de pesquisa; amostragens e técnicas de pesquisa; elaboração, análise e interpretação de dados. São Paulo: Atlas.

18. Magnani Parra, G.I.A. (2018) Migração Atlética de Jogadores de Futebol Brasileiro para o Exterior: O processo de adaptação cultural (Tese de doutorado). Universidade São Judas Tadeu, USJT, São Paulo, SP, Brasil. Disponível em: https://www.usjt.br/biblioteca/mono_disser/mono _diss/2019/451.pdf. Acesso em: 12 set. 2020.

19. Ruiz, O. J. I., Ispizua, M. A. (1989). La descodificacion de la vida cotidiana: metodos de investigacion cualitativa: Universidad de deusto Bilbao.

20. Oyserman, D., Elmore, K., Smith G. (2012). Self, self-concept, and identity. Tangney, J., Leary, M. (Eds.), Handbook of Self and Identity, 69-104, New York, NY: Guilford Press.

21. Oliveira, H. Z., Dias, T. S., Sabino, B. C. R., Dias, C., Corte-Real, N., Fonseca, A. M. (2019). Adaptação transcultural do Empowering Disempowering Motivational Climate Questionnaire para a língua portuguesa e análise da invariância em atletas masculinos brasileiros e portugueses. Cuadernos de Psicología del Deporte, 19(1), 1-18. https://doi.org/10.6018/cpd.335451.

22. Occhino, J. L., Mallett, C. J., Rynne, S. B., Carlisle, K. N. (2014). Autonomy-supportive pedagogical approach to sports coaching: Research, challenges and opportunities. International Journal of Sports Science \& Coaching, 9(2), 401-415. https://doi.org/10.1260/1747-9541.9.2.401.

23. Pilkington, G., Hounsome, J. (2017). Planning and managing my Review. Boland, A., Cherry, G., Dickson, R. (Eds.), Doing a Systematic Review, 21-42. London, UK: Sage.

24. Ribeiro, C. H. D. V., Dimeo, P. (2009) The experience of migration for Brazilian football players. Sport in Society, 12(6), 725-736. https://doi.org/10.1080/17430430902944519.

25. Ryba, T. V., Haapanen, S., Mosek, S., N, K. (2012). Towards a conceptual understanding of acute cultural adaptation: A preliminary examination of ACA in female swimming. Qualitative research in sport, exercise and health, 


\section{Souza, Miranda, Correa, Junior \& Brandão}

4(1),
https://doi.org/10.1080/2159676X.2011.653498.

26. Ryba, T. V., Schinke, R. J., Stambulova, N. B., Elbe, A.M. (2017). ISSP position stand: Transnationalism, mobility, and acculturation in and through sport. International Journal of Sport and Exercise Psychology, 16(5), 520-534. DOI: https://doi.org/10.1080/1612197X.2017.1280836.

27. Ryba, T. V., Stambulova, N. B., Ronkainen, N. J. (2016). The work of cultural transition: An emerging model. Frontiers in Psychology, 7, (427).

https://doi.org/10.3389/fpsyg.2016.00427.

28. Santos, C. M. C., Pimenta, C. A.M., Nobre, M. R. C. (2007) A Estratégia PICO para a construção da pergunta de pesquisa e busca de evidências. Epidemiologia e Serviços de Saúde, 23(1), 183184. https://doi.org/10.1590/S0104116920070003023.

29. Schinke, R. J., McGannon, K. R., Battochio, R. C., Wells, G. D. (2013). Acculturation in elite sport: a thematic analysis of immigrant athletes and coaches. Journal of sports sciences, 31(15), 16761686.

https://doi.org/10.1080/02640414.2013.794949.

30. Schinke, R. J., Michel, G., Gauthier, A. P., Pickard, P., Danielson, R., Peltier, D., Peltier, M. (2006). The adaptation to the mainstream in elite sport: A Canadian Aboriginal perspective. The sport psychologist, 20(4), 435-448. https://doi.org/10.1123/tsp.20.4.435.

31. Schinke, R. J., Yukelson, D., Bartolacci, G., Battochio, R. C., Johnstone, K. (2011). The challenges encountered by immigrated elite athletes. Journal of Sport Psychology in Action, 2(1), 10-20. https://doi.org/10.1080/21520704.2011.556179.

32. Schwartz, S. J., Unger, J. B., Zamboanga, B. L., Szapocznik, J. (2010). Rethinking the concept of acculturation: implications for theory and research. American Psychologist, 65(4), 237-251: https://doi.org/10.1037/a0019330.

33. Stoltenburg, A. L., Kamphoff, C. S., Bremer, K. L. (2011). Transitioning out of sport: the psychosocial effects of collegiate athletes' careerending injuries. Athletic Insight Journal, 3(2), 115-133.

34. Stone P. W. (2004). Popping the (PICO) question in research and evidence-based practice. Applied
Nursing Research, 15(3):197-198. https://doi.org/10.1053/apnr.2002.34181.

35. Soler, M. (2020) Orientaciones psicológicas para el deportista «migrante» Saber Competir, Revista de Psicologia Del Deporte, (3). Disponível em: https://sabercompetir.com/deportista-migrante/. Acesso em: 25 set. 2020.

36. Tertuliano, I. W., Machado, A. A., Deutsch, S., Montiel, J. M., Bartholomeu, D. (2018). Motivos e intenções para expatriação de voleibolistas. Revista de Administração Contemporânea, 22(4), 531-551.

https://doi.org/10.1590/19827849rac2018170032.

37. Tretyakevich, N., Las Heras, M., Hall, D. T. (2015). Expatriation: work-life balance and psychological success. Balance, Technology, and Globalization IESE Business School, Barcelona.

38. Vasconcellos Ribeiro, C. H., Dimeo, P. (2009). The experience of migration for Brazilian football players. Sport in Society, 12(6), 725-736. https://doi.org/10.1080/17430430902944159.

39. Ward, C., Kennedy, A. (1999). The measurement of sociocultural adaptation. International journal of intercultural relations, 23(4), 659-677. https://doi.org/10.1016/S0147-1767(99)00014-0.

40. Yamakawa, E. K., Kubota, F. I., Beuren, F. H., Scalvenzi, L., Miguel, P. A. C. (2014). Comparativo dos softwares de gerenciamento de referências bibliográficas: Mendeley, EndNote e Zotero. Transinformação, 26(2), 167-176. https://doi.org/10.1590/01033786201400020006.

41. Yukelson, D., Carlson, M. (1996). Stress and its impact on intercollegiate student athletes. Inaugural NCAA Champs Life Skills Manual. Overland Park, KS: National Collegiate Athletic Association. 


\section{Expatriação de atletas e seus aspectos psicológicos}

Tabela 2 Resultados descritivos dos participantes dos estudos.

\begin{tabular}{|c|c|c|c|c|c|c|c|}
\hline Autores & Ano & Periódico & Objetivo & $\begin{array}{c}\text { Participantes } \\
\text { (atletas) }\end{array}$ & $\begin{array}{l}\text { Idade } \\
\text { (anos) }\end{array}$ & $\begin{array}{c}\text { Modalidade } \\
\text { Esportiva }\end{array}$ & $\begin{array}{c}\text { Instrumento } \\
\text { Utilizado }\end{array}$ \\
\hline $\begin{array}{l}\text { Schinke, } \\
\text { Michel, } \\
\text { Gauthier, } \\
\text { Pickard e } \\
\text { Danielson }\end{array}$ & 2006 & $\begin{array}{l}\text { The Sport } \\
\text { Psychologist }\end{array}$ & $\begin{array}{l}\text { Delinear os desafios e estratégias de } \\
\text { adaptação dos atletas aborígines durante } \\
\text { seus anos no esporte de elite. }\end{array}$ & $\begin{array}{l}16 \text { homens e } \\
7 \text { mulheres }\end{array}$ & $17 \pm 42$ & $\begin{array}{l}\text { Hóquei no gelo, } \\
\text { lacrosse, boxe, } \\
\text { atletismo, futebol, } \\
\text { tae kwan e corrida } \\
\text { de cross country }\end{array}$ & $\begin{array}{c}\text { Entrevista } \\
\text { semiestruturada }\end{array}$ \\
\hline $\begin{array}{l}\text { Ryba, } \\
\text { Haapanen, } \\
\text { Mosek e } \\
\text { Kwok Ng }\end{array}$ & 2012 & $\begin{array}{l}\text { Qualitative } \\
\text { Research in } \\
\text { Sport, Exercise } \\
\text { and Health }\end{array}$ & $\begin{array}{l}\text { Pesquisar e conceituar a adaptação cultural } \\
\text { aguda em atletas nadadoras da Finlândia } \\
\text { durante o acampamento de treinamento na } \\
\text { Austrália. }\end{array}$ & 6 mulheres & $17 \pm 26$ & Natação & $\begin{array}{c}\text { Entrevista } \\
\text { semiestruturada }\end{array}$ \\
\hline $\begin{array}{l}\text { Schinke, } \\
\text { McGannon, } \\
\text { Battochio e } \\
\text { Wells }\end{array}$ & 2013 & $\begin{array}{l}\text { Journal of Sports } \\
\text { Sciences }\end{array}$ & $\begin{array}{l}\text { Identificar questões-chave relacionadas à } \\
\text { aculturação de atletas de rendimento } \\
\text { imigrantes no Canadá. }\end{array}$ & $\begin{array}{l}10 \text { homens e } \\
3 \text { mulheres }\end{array}$ & $16 \pm 32$ & $\begin{array}{l}\text { Hóquei no gelo, } \\
\text { natação, boxe, } \\
\text { nado sincronizado } \\
\text { e basquetebol }\end{array}$ & $\begin{array}{c}\text { Entrevista } \\
\text { semiestruturada }\end{array}$ \\
\hline $\begin{array}{l}\text { Agergaard e } \\
\text { Ryba }\end{array}$ & 2014 & $\begin{array}{l}\text { Sociology of } \\
\text { Sport Journal }\end{array}$ & $\begin{array}{l}\text { Contextualizar e discutir argumentos para } \\
\text { o desenvolvimento de um quadro } \\
\text { interdisciplinar para explicar as } \\
\text { experiências vividas do estreitamento } \\
\text { entre migração transnacional e } \\
\text { desenvolvimento de carreira no esporte } \\
\text { profissional. }\end{array}$ & 18 mulheres & $23 \pm 24$ & Futebol & $\begin{array}{c}\text { Entrevista } \\
\text { semiestruturada }\end{array}$ \\
\hline \multirow[t]{2}{*}{$\begin{array}{l}\text { Ryba, } \\
\text { Stambulova e } \\
\text { Ronkainen }\end{array}$} & 2016 & $\begin{array}{l}\text { Frontiers in } \\
\text { Psychology }\end{array}$ & $\begin{array}{l}\text { Identificar as tarefas de desenvolvimento } \\
\text { de transições culturais e estratégias / } \\
\text { mecanismos, através dos quais as } \\
\text { transições culturais foram realizadas. }\end{array}$ & $\begin{array}{l}7 \text { homens e } \\
8 \text { mulheres }\end{array}$ & $18 \pm 37$ & $\begin{array}{l}\text { Hóquei no gelo, } \\
\text { basquetebol, } \\
\text { futebol, handebol } \\
\text { e arco e flecha }\end{array}$ & $\begin{array}{c}\text { Entrevista } \\
\text { semiestruturada }\end{array}$ \\
\hline & 2016 & $\begin{array}{l}\text { Inquiries in Sport } \\
\text { \& Physical } \\
\text { Education }\end{array}$ & $\begin{array}{l}\text { Aprofundar sobre casos de migração de } \\
\text { mão-de-obra esportiva e identidade do } \\
\text { atleta e aprender com atletas em migração }\end{array}$ & 2 homens & $31 \pm 39$ & Ciclismo & $\begin{array}{c}\text { Entrevista não } \\
\text { estruturada }\end{array}$ \\
\hline
\end{tabular}


Souza, Miranda, Correa, Junior \& Brandão

\begin{tabular}{|c|c|c|c|c|c|c|c|}
\hline $\begin{array}{l}\text { Diakaki, } \\
\text { Chroni e } \\
\text { Goudas }\end{array}$ & & & $\begin{array}{l}\text { sobre os mecanismos narrativos utilizados } \\
\text { para reconstruir suas prioridades e } \\
\text { identidades. }\end{array}$ & & & & \\
\hline Magnani & 2018 & $\begin{array}{c}\text { CAPES/MEC } \\
\text { Tese }\end{array}$ & $\begin{array}{l}\text { Mapear o processo de adaptação cultural } \\
\text { de jogadores brasileiros de futebol } \\
\text { profissional em situação de migração } \\
\text { atlética, à luz do Modelo de Transição } \\
\text { Cultural. }\end{array}$ & 23 homens & $19 \pm 34$ & Futebol & $\begin{array}{c}\text { Entrevista } \\
\text { semiestruturada }\end{array}$ \\
\hline Lofredo & 2018 & $\begin{array}{l}\text { Periódicos } \\
\text { CAPES/MEC } \\
\text { Dissertação }\end{array}$ & $\begin{array}{l}\text { Compreender como uma atleta de elite do } \\
\text { handebol feminino brasileiro vivenciou o } \\
\text { processo de migração atlética } \\
\text { internacional e analisá-lo à luz do modelo } \\
\text { de transição cultural de Ryba, Stambulova } \\
\text { e Ronkainen (2016). }\end{array}$ & 1 mulher & 39 & Handebol & $\begin{array}{c}\text { Entrevista } \\
\text { semiestruturada }\end{array}$ \\
\hline
\end{tabular}

Tabela 3 Resultados analíticos dos estudos.

\section{Resultados}

Conclusão

Indicações de que os atletas se envolvem em dois tipos de adaptação: a auto adaptação e o ambiente adaptado.
Desenvolvimento de estratégias pessoais e contextuais antes e durante o ingresso no esporte de elite.

As estratégias de coaching podem ser eficazes e facilitadoras.

Os modelos podem ser integrados como um recurso eficaz nas possíveis estratégias de adaptação.

Espera-se que os pesquisadores da psicologia do esporte, interessados nas práticas de adaptação cultural, busquem investigar os desafios pessoais vivenciados por atletas minoritários.

Acredita-se que uma forte parceria da prática aplicada, informada pela pesquisa aplicada em psicologia do esporte, contribuirá para a adaptação positiva e excelência esportiva de atletas aborígines canadenses. 


\section{Expatriação de atletas e seus aspectos psicológicos}

Os participantes mantiveram seu bem-estar psicológico, negociando as práticas cotidianas, por meio do envolvimento com o novo 2 contexto cultural.

A adaptação cultural aguda foi mediada em dois níveis: o relacionamento da equipe e o novo contexto cultural.

Dois temas centrais foram identificados: A navegação por duas 3 visões de mundo: a aculturação e as cargas de aculturação.

O recrutamento transnacional utiliza redes sociais, agências

4 individuais e o desenvolvimento de relações com jogadores e treinadores.

$\mathrm{O}$ estabelecimento como atleta transnacional, está conectado à adaptação cultural e psicológica, bem como ao desenvolvimento do pertencimento transnacional.

O término da carreira atlética profissional está conectado a uma (re) constituição da rede transnacional e ao sentimento de pertencimento.

Três mecanismos subjacentes do processo de transição ajudaram a

5 adaptabilidade da carreira atlética, o reposicionamento social, a negociação de práticas culturais e a reconstrução de significados. A análise dos dados propõe um modelo temporal de transição cultural.

As deslocações físicas e mentais dos ciclistas desencadearam e intensificaram processos de negociação de identidade, formação e reconstrução.

6 Uma contínua negociação ocorreu entre o eu, a sociedade e a cultura esportiva e os atletas lutaram para encontrar a sua identidade dentro dos estereótipos das histórias de sucesso esportivo aceitos.
O relacionamento é um espaço cultural discursivo e ponto de partida para examinar o papel da cultura nas funções psicológicas na migração de curto prazo.

Direcionar o estudo da adaptação nos processos e não nos resultados, para uma compreensão mais sutil da adaptação cultural por meio de metodologias qualitativas para aprofundar essa área de estudo.

Aprofundar-se nas investigações, utilizando estudos de caso para fornecer uma descrição holística do processo de aculturação, inserindo todas as pessoas envolvidas no contexto esportivo.

O desenvolvimento esportivo profissional não pode ser estudado apenas a partir do ponto de vista do nacionalismo metodológico. Os estudos devem incluir os diferentes contextos em que os atletas desenvolvem o seu trabalho esportivo e a influência da mobilidade transnacional e adaptação.

A pesquisa forneceu aos profissionais que trabalham nas áreas de aconselhamento de carreira e suporte migratório, conteúdos para aprimorar as adaptabilidades e o bem-estar psicológico dos atletas migrantes.

Enquanto os atletas viviam uma vida em ambientes e espaços sociais diferentes e desconhecidos, eles tentaram encontrar coerência através e dentro das suas próprias histórias do passado.

O enriquecimento dos recursos narrativos pela aceitação e adoção de diversas narrativas alternativas, ajudará as intervenções de integração e a transição positiva nas diferentes fases da carreira de um atleta.

Os atletas devem ter a oportunidade de recorrer a um rico repositório de esquemas narrativos para refletir, relacionar e problematizar.

Os repertórios narrativos podem incentivar e inspirar outros atletas a compartilhar seus pensamentos íntimos sobre experiências, promover o senso de pertencimento e, assim, construir um senso saudável de si e preservar o bem-estar psicológico.

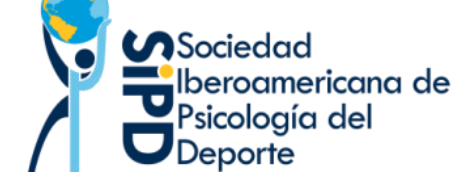


Souza, Miranda, Correa, Junior \& Brandão

Não só os atletas, mas também os treinadores e os praticantes de psicologia

desportiva podem se beneficiar de programas de promoção profissional sensíveis à cultura e, ao longo da vida, que reforçam o uso de narrativas.

Os consultores em psicologia do esporte precisam se sensibilizar a respeito do impacto das experiências de migração nas identidades dos atletas, a fim de atuarem como um canal de comunicação entre eles e o novo ambiente, para facilitar uma adaptação suave nos novos contextos, cuidando do ser humano e não apenas do fazer humano.

Os atletas evidenciaram o idioma, o clima, a distância da família e Fatores emocionais aliados a influência dos meios de comunicação tiveram grande

7 os diferentes costumes culturais e econômicos como as principais

impacto no processo de adaptação cultural dos atletas. dificuldades relacionadas ao processo de adaptação cultural.

Na fase de pré-transição, a atleta não foi preparada para enfrentar a migração atlética internacional.

Os principais pontos que poderiam dificultar o processo de aculturação na migração atlética são: informações sobre o futuro país/clube, idioma, apoio e discriminação

8 Na fase de preparação cultural aguda, ficaram evidentes questões social, distância da família e diferenças culturais. relativas ao choque cultural.

$\mathrm{Na}$ fase de adaptação sociocultural, a atleta se mostrou mais bem ajustada aos novos ambientes e superou algumas das dificuldades experimentadas na fase anterior. 